\title{
Modulating transmission properties of nanoscale transistors by dipole effects near contacts
}

\author{
Li Yang, Jian Wu*, Wenhui Duan, and Bing-Lin Gu \\ Department of Physics and Center for Advanced Study, \\ Tsinghua University, Beijing 100084, People's Republic of China
}

(Dated: February 7, 2019)

\begin{abstract}
We theoretically demonstrate that a dipole layer on the electrode can modulate the transmission properties of nanoscale devices by influencing the contact properties, through first principles simulations on carbon nanotube based field effect transistors. The dipole layer is realized by potassium adsorption on $\mathrm{Au}$ electrodes, which shifts the electrostatic potential at the near contact region significantly. The dipoles parallel to the direction of the bias voltage may act as a supplement to the effect of gate voltages, while the perpendicular dipoles may modify the interface barrier of the contacts.
\end{abstract}

PACS numbers: 85.35.-p, 73.40.Cg, 73.22.-f, 85.35.Kt

\footnotetext{
* Author to whom any correspondence should be addressed. Email address: wu@castu.tsinghua.edu.cn
} 


\section{(a)}

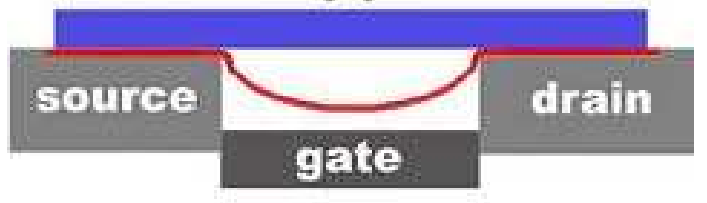

\section{(b)}

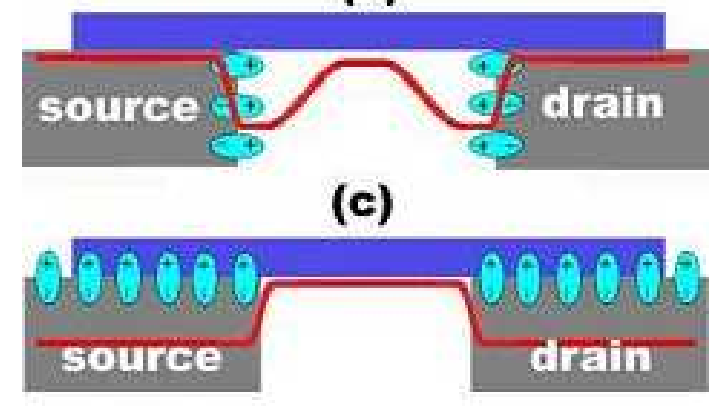

FIG. 1: (Color online) Sketch of the electrostatic potential (the solid lines) on the sample induced by (a) a gate voltage, (b) "parallel" dipoles, and (c) "perpendicular" dipoles.

The miniaturization of devices is an irresistible trend in industrial manufacture and becomes a hot research topic. Nanoscale devices, such as field-effect transistors (FETs) based on nanotubes [1, 2] or nanowires [3], have attracted more and more interests. In general, the transmission properties of FETs are controlled through a gate voltage. However, with the device size decreasing to nanoscale, the potential profile near the electrodes may play a central role in electron transport behavior, especially for systems with metal-semiconductor contacts. In such systems, the potential pinning effect of the metallic electrodes will lead to a change of the potential profile near the contacts as shown in Fig. 1(a) 4, 5] under the gate voltage, which is not favorable for the quantum tunneling of electrons, and thus make the conventional modulation by the gate voltage less effective. Though it has been demonstrated that the device performance may be improved by using needle-like contacts [4] or using small gate segments near the electrodes [5] or adopting special gate configuration [6], the problem is only partially solved since the potential pinning effect of the metallic electrodes can not be fully overcome but only limited to a slightly smaller region. Considering the fact that the potential pinning effect comes from the metallic surface, modulating the property of the metallic surface directly might be a better approach to improve the performance of nanoscale devices.

In this letter, we demonstrate that the modulation of the transmission properties of 
nanoscale transistors can be realized by introducing a dipole layer on the electrodes. As schematically shown in Fig. 1(b), an additional electrostatic potential induced by a dipole layer on the lateral side of electrodes may significantly change the electrostatic potential of the functional body near the interface, and acts as a supplement to the effect of gates. With the potential pinning effect of the metallic surface balanced or strengthened by the dipole layer, a significant modulation effect can be expected in the system. Besides, the dipole layer on the top side of electrodes will modify the electrostatic potential of the functional body at the interface region as shown in Fig. 1(c) and thus modify the interface barrier.

In general, the dipoles parallel to and perpendicular to the direction of the bias voltage have very different effects on the system. The "parallel" dipoles may act as a supplement to the effect of gates; while the "perpendicular" dipoles may only modify the interface barrier of the contacts. As a case study, we will investigate the effect of the dipole layer, which is induced by chemical adsorption on electrodes, on the contact properties of carbon nanotube based field effect transistors (CNTFETs). It is known that adsorption of alkali atoms on metal surface will lead to electric dipole moments [7, 8, 9]. Here, we will focus on the case of $\mathrm{K}$ adsorption on Au electrodes in CNTFET, which has also been extensively studied in recent experiments [4, 10, 11]. Specifically, we use the lateral adsorption and top adsorption (as shown in Fig. 2) to introduce "parallel" dipoles and "perpendicular" dipoles respectively.

Our study is performed by numerical simulations using the plane wave basis VASP code 12, 13] within the framework of density functional theory (DFT) under local density approximation (LDA). The planewave cutoff energy is $287 \mathrm{eV}$. Integration over the Brillouin zone is done using $\Gamma$ centered Monkhorst-Pack scheme [14] with $1 \times 1 \times 1$ and $2 \times 8 \times 1$ for lateral and top adsorption respectively. Relaxations of atomic positions were carried out until the forces on each atom are less than $0.01 \mathrm{eV} / \AA$.

The K-adsorbed Au/CNT contact system studied is represented by the supercell as shown in Figs. 2(b) (lateral adsorption) and 2(d) (top adsorption) with periodic boundary condition, where a large enough vacuum layer and dipole corrections [15] in the z direction are introduced to avoid non-physical interactions between repeated images of the unit cell. $(8,0)$ carbon nanotube and $\mathrm{Au}(100)$ slab are used in our simulations. Test calculations are done with both five and three $\mathrm{Au}$ (100) layers and no essential difference is found in the properties concerned. Therefore, we take the slab model of three $\mathrm{Au}(100)$ layers in the following discussion for both lateral and top adsorption cases. 


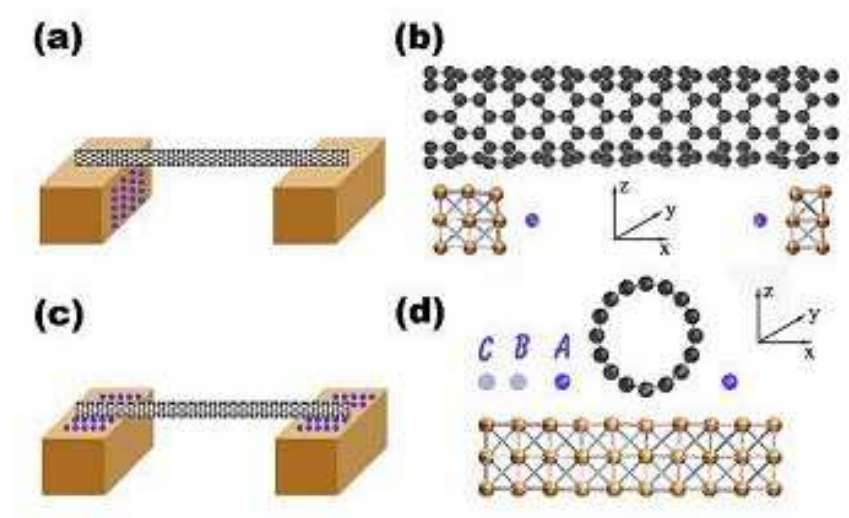

FIG. 2: Schematic illustration (a) and atomic geometry (b) of $\operatorname{Au}(100) / \mathrm{CNT}(8,0)$ contact with K lateral adsorption. Schematic illustration (c) and atomic geometry (d) of $\mathrm{Au}(100) / \mathrm{CNT}(8,0)$ contact with $\mathrm{K}$ top adsorption. The gold, purple, and black balls represent $\mathrm{Au}, \mathrm{K}$, and $\mathrm{C}$ atoms, respectively.

In most recent FET experiments, annealing is done before the $\mathrm{K}$ adsorption process to obtain intimate contacts between electrodes and the nanotube [16, 17]. So, as the first step of our simulation, a good contact between Au electrodes and CNT is obtained by a structural optimization with the bottom layer of the $\mathrm{Au}$ slab fixed and all other $\mathrm{Au}$ atoms and $\mathrm{C}$ atoms fully relaxed. By comparing the binding energy for typical contact geometries such as DT (direct on top), BM (bridge middle), and HC (hexagonal center), we obtain the most stable geometry (BM geometry) of $\mathrm{Au} / \mathrm{CNT}$ contact. Here the lattice parameters of $\mathrm{Au}(100) \mathrm{slab}$ along the tube axial direction are elongated slightly to match that of $(8,0)$ CNT while those in the other two directions are equally compressed to make the volume a constant as done in Ref. [18].

Then we determine the contact structures associated with $\mathrm{K}$ adsorption. It is found that $\mathrm{K}$ atoms can be chemically adsorbed on clean $\mathrm{Au}(100)$ surface, with adsorption energy of up to $2.86 \mathrm{eV}$ (at the hollow sites), which is much larger than that on CNT (typically, 1.43 $\mathrm{eV}$ ). This indicates that $\mathrm{K}$ atoms prefer to be adsorbed on the electrodes rather than on the CNT during K doping treatment in CNTFET experiments. For obtaining the favorable adsorption site of $\mathrm{K}$ atom on $\mathrm{Au}$ surface in the $\mathrm{Au} / \mathrm{CNTFET}$ system, the $\mathrm{K}$ atom is initially put on different hollow sites with different distance from the CNT and then the structures are optimized. The adsorption energies obtained are $3.04 \mathrm{eV}, 2.81 \mathrm{eV}$ and $2.79 \mathrm{eV}$, respectively, for the three adsorption sites A, B and C (as shown in Fig. 2(d)). This means that the K 

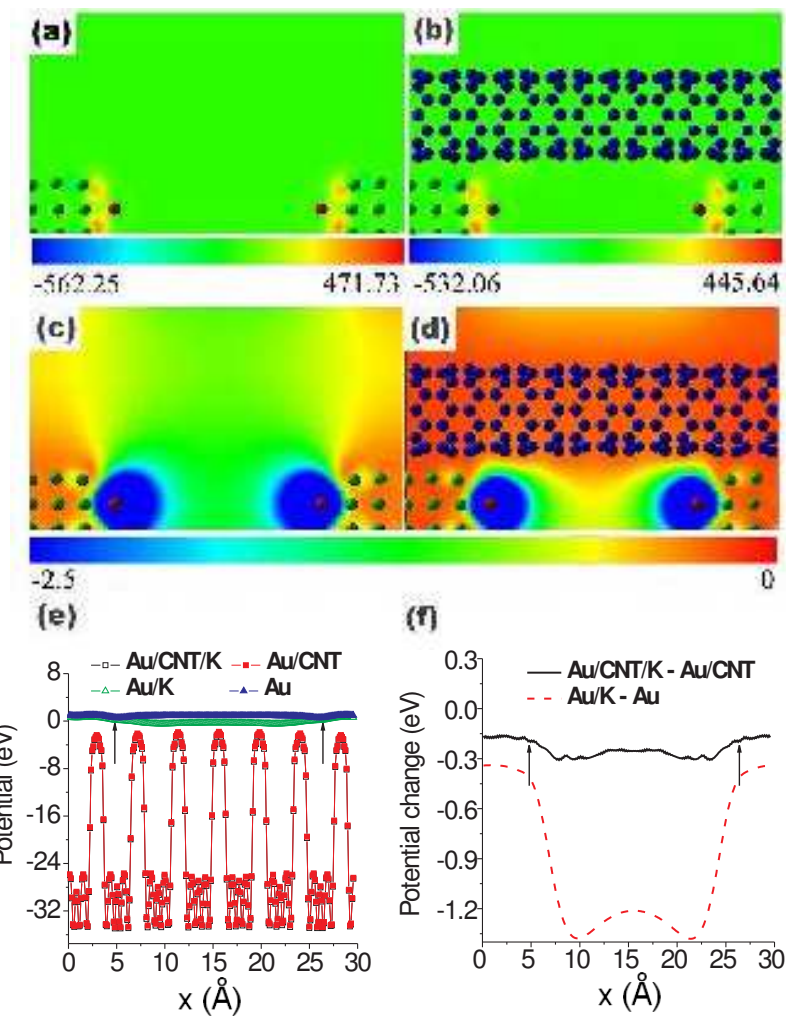

(f)

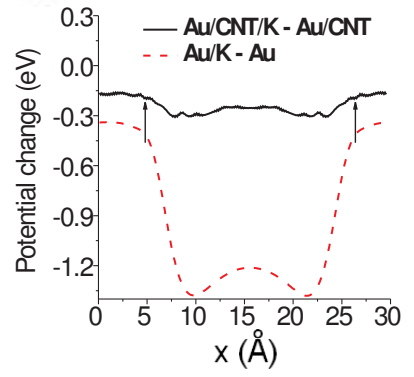

FIG. 3: (Color online) The physical property changes induced by K lateral adsorption. (a) Charge difference, and (c) Local potential difference between clean and K adsorbed Au surfaces. (b) Charge difference, and (d) Local potential difference between clean and $\mathrm{K}$ adsorbed Au/CNT contacts. (All the slices are selected passing through the K atoms.) (e) Local potential along the line perpendicular to the lateral surface and passing through the bottom carbon atoms of CNT for the four systems: clean Au surface, $\mathrm{K}$ adsorbed $\mathrm{Au}$ surface, pure Au/CNT contact, and $\mathrm{K}$ adsorbed $\mathrm{Au} / \mathrm{CNT}$ contact. (f) Local potential change after $\mathrm{K}$ adsorption along the same line, for $\mathrm{Au}$ surface as well as $\mathrm{Au} / \mathrm{CNT}$ contact. The green, red, and blue balls represent $\mathrm{Au}, \mathrm{K}$, and $\mathrm{C}$ atoms respectively. The solid arrows denote the edges of Au electrodes.

atom prefers to stay at $\mathrm{A}$ site closest to the CNT. In the optimized structure, the $\mathrm{K}$ atom also deviates from the center of the hollow site and moves towards to the CNT. Therefore, in the following simulations, we focus on the structures shown in Figs. 2(b) and 2(d), where the $\mathrm{K}$ atoms are placed around the hollow sites of $\mathrm{Au}(100)$ surface near the $(8,0) \mathrm{CNT}$, to demonstrate the dipole effects.

The lateral adsorption and top adsorption lead to "parallel" dipoles and "perpendicular" dipoles, respectively. First we discuss the lateral adsorption case. To clearly show the effect 
of a dipole layer on a device, we study the effect of a pure dipole layer by taking away the CNT from the system shown in Fig. 2(b). Fig. 3(a) shows the charge transfer in this case. It is obvious that almost all the valence electrons of the $\mathrm{K}$ atoms transfer to the Au surface, and this results in dipoles pointing from the surface to the $\mathrm{K}$ ions. Note that the dipoles form a dipole layer due to the periodical boundary condition used in the simulation, which is just what we want. Such a dipole layer on electrodes will cause a downward shift of the electrostatic potential nearby, as shown in Fig. 3(c). For a better view, the local potential plotted along the line perpendicular to the lateral surface and passing through the bottom carbon atom sites of CNT is shown in Fig. 3(e) and the potential change is shown in Fig. $3(\mathrm{f})$. It can be seen that the downward shift of potential is up to $1.37 \mathrm{eV}$, a large value for most devices.

For a dipole layer of finite size (with characteristic length $L$ ), the potential shift induced by the dipole layer at a distance $x$ is nearly a constant when $x \ll L$, since the dipole layer can be regarded to be infinitely extended in this case. While the potential shift decreases as $x^{-2}$ when $x \gg L$, where the dipole layer can be regarded as a point dipole. Furthermore, the characteristics of the dipole layer can be modulated by changing its dipole strength and the layer size. For adsorption induced dipoles in CNTFET systems, this can be realized by changing the adsorption coverage and electrodes size. Thus, the potential drop caused by the dipole layer in the near interface region may be used to modulate the transmission properties of a nanoscale device, as a supplement to the effect of gates. Moreover, the change of the potential profile (schematically shown in Figs. 1(a) and 1(b)) is now limited to a length of approximately $5 \AA$ (see Fig. 3(f)), much smaller than the typical length (about $5 \mathrm{~nm}$ ) under a gate voltage about $10 \mathrm{~V}$ [4]. Therefore, such a supplement is quite effective.

Next, we quantitatively show the effect of the potential shift on the CNT induced by the dipole layer. When a CNT is included in the system, most of the valence electrons of $\mathrm{K}$ atoms transfer to the Au surface while little to the CNT (as shown in Fig. 3(b)). Consequently, the dipole layer formed between $\mathrm{K}$ ions and the Au surface still plays the main role. This dipole layer will introduce an additional electrostatic potential as discussed above, and, at the same time, cause a charge redistribution between Au electrodes and the CNT. This charge redistribution counteracts the effect of the dipole layer and reduces the potential change on the CNT (as shown in Fig. 3(d)). Fig. 3(e) shows the local potential on the CNT along the line perpendicular to the lateral surface and passing through the bottom carbon 
atoms of the CNT, and Fig. 3(f) shows the related potential change. In comparison with the case of the pure dipole layer, the charge redistribution reduces the potential change effect, but the net effect of the dipole layer on the CNT is still found to be a downward potential shift up to $0.37 \mathrm{eV}$, indicating a significant change of contact properties. It should be noted that this effect will be more significant with larger coverage ratio of K atoms. Furthermore, the potential shift on the CNT is somewhat underestimated since the electrostatic response from other CNTs is included in our simulations due to the periodical boundary condition, while only one or very few CNTs are presented in most real systems. Therefore, through our calculation, we demonstrate that "parallel" dipoles on electrodes can effectively complement the conventional effect of gate voltage; such complement can significantly change the contact properties and thus modify the transmission properties of CNTFETs.
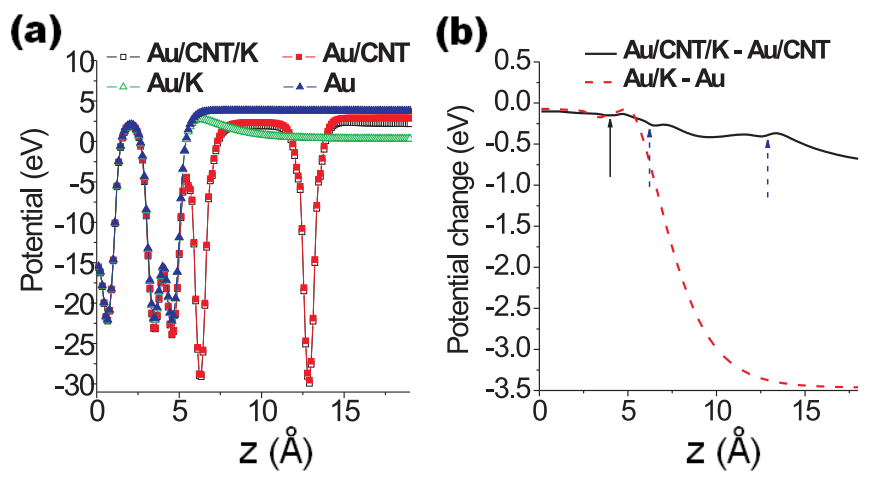

FIG. 4: (Color online) The physical property changes induced by K top adsorption. (a) Local potential along the line perpendicular to the top surface and passing through the bottom carbon atoms of CNT for the four systems: clean Au surface, $\mathrm{K}$ adsorbed Au top surface, pure Au/CNT contact, and $\mathrm{K}$ adsorbed on top surface of $\mathrm{Au} / \mathrm{CNT}$ contact. (b) Local potential difference along the same line, for $\mathrm{Au}$ surface and $\mathrm{Au} / \mathrm{CNT}$ contact. The solid arrow denotes the edge of $\mathrm{Au}$ electrode, and the dashed arrows denote the edges of CNT.

Although both the top surface adsorption and the lateral adsorption result in the dipole layer, they have quite different effects on the device. Similar to the lateral adsorption case, the adsorption of $\mathrm{K}$ atoms on $\mathrm{Au}$ top surface will result in a dipole layer formed by dipoles pointing from the surface to the $\mathrm{K}$ ions, and thus cause a downward shift of the electrostatic potential. Such additional potential will lead to a charge redistribution between Au electrode and the CNT, which counteracts the potential change and reduces the potential change on 
the CNT.

Fig. 4 shows the local potential and its change induced by $\mathrm{K}$ adsorption for Au surface and $\mathrm{Au} / \mathrm{CNT}$ contact system with $\mathrm{K}$ top adsorption. Here, the potential is plotted along the line perpendicular to the top surface and passing through the bottom carbon atom of CNT. It is found that the potential on the CNT is greatly shifted: the potential of the bottom carbon atoms of CNT is downward shifted up to $0.25 \mathrm{~V}$ and the potential of the topmost carbon atoms of CNT is downward shifted up to $0.39 \mathrm{~V}$. This represents the magnitude of interface barrier (between the metal and the CNT) modification, and might be underestimated for the same reason as we discussed in the lateral adsorption case. Therefore, "perpendicular" dipoles may also contribute to the transmission modulation of nanoscale transistors by modifying the interface barrier. It should also be noted that due to the fact that effect of the dipole layers is orientational dependent, it can only affect the interface barrier perpendicular to the plane of the dipole layer.

In conclusion, we have demonstrated that introducing dipole layers on the electrodes can significantly modulate the contact properties of nanoscale devices. Specifically, via $\mathrm{K}$ adsorption on $\mathrm{Au}$ electrodes, we introduced dipole layers parallel or perpendicular to the direction of the bias voltage, and quantitatively revealed their effects on the contact properties of CNTFETs. The "parallel" dipoles induce a change of the electrostatic potential of the functional body (especially near the contact), as a supplement to the effect of gates; while the "perpendicular" dipoles modify the interface barrier of the contacts. Such striking effects of the dipole layer might play an important role in optimizing contact properties in the design of nanoelectronic devices.

This work was supported by National Natural Science Foundation of China (Grant No. 10325415 and 10274038), the Ministry of Science and Technology of China (Grant No. 2002AA311153), and the Ministry of Education of China (Grant No. 200017 and NCET04-0085).

[1] S. Tans, A. R. Vershueren, and C. Dekker, Nature (London) 393, 49 (1998).

[2] R. Martel, T. Schmidt, H. R. Shea, T. Hertel, and Ph. Avouris, Appl. Phys. Lett. 73, 2447 (1998). 
[3] Y. Cui and C. M. Lieber, Science 291, 851 (2001).

[4] S. Heinze, J. Tersoff, R. Martel, V. Derycke, J. Appenzeller, and Ph. Avouris, Phys. Rev. Lett. 89, 106801 (2002).

[5] S. J. Wind, J. Appenzeller, and Ph. Avouris, Phys. Rev. Lett. 91, 058301 (2003).

[6] S. Heinze, J. Tersoff, and Ph. Avouris, Appl. Phys. Lett. 83, 5038 (2003).

[7] Physics and Chemistry of Alkali Adsorption, edited by H. P. Bonzel, A. M. Bradshaw, and G. Ertl (Elsevier, Amsterdam, 1989).

[8] H. D. Ebinger, H. J. Jänsch, C. Polenz, B. Polivka, W. Preyss, V. Saier, R. Veith, and D. Fick, Phys. Rev. Lett. 76, 656 (1996).

[9] G. Rangelov, and L. Surnev, Surf. Sci. 185, 457 (1987).

[10] V. Derycke, R. Martel, J. Appenzeller, and Ph. Avouris, Appl. Phys. Lett. 80, 2773 (2002).

[11] M. Radosavljevic, J. Appenzeller, and Ph. Avouris, Appl. Phys. Lett. 84, 3693 (2004).

[12] See, for example, G. Kresse and J. Furthmüller, Comput. Mater. Sci. 6, 15 (1996).

[13] D. Vanderbilt, Phys. Rev. B 41, R7892 (1990).

[14] H. J. Monkhorst and J. D. Pack, Phys. Rev. B 13, 5188 (1976).

[15] J. Neugebauer, and M. Scheffler, Phys. Rev. B 46, 16067 (1992).

[16] S. Auvray, J. Borghetti, M. F. Goffman, A. Filoramo, V. Derycke, J. P. Bourgoin, and O. Jost, Appl. Phys. Lett. 84, 5106 (2004).

[17] Y. Yaish, J.-Y. Park, S. Rosenblatt, V. Sazonova, M. Brink, and P. L. McEuen, Phys. Rev. Lett. 92, 046401 (2004).

[18] V. G. Zavodinskya, and I. A. Kuyanov, J. Appl. Phys. 81, 2715 (1997). 